\author{
Asian Journal of \\ Medical and Biological Research \\ ISSN 2411-4472 (Print) 2412-5571 (Online) \\ www.ebupress.com/journal/ajmbr
}

\title{
Article \\ Interleukin-33, an interleukin-1 like cytokine accelerates cell migration in IEC-6 cells
}

\author{
Md. Shafiqul Islam ${ }^{1,2, *}$, Masatoshi Hori $^{2}$ and Hiroshi Ozaki ${ }^{2}$ \\ ${ }^{1}$ Department of Pharmacology, Faculty of Veterinary Science, Bangladesh Agricultural University, \\ Mymensingh-2202, Bangladesh \\ ${ }^{2}$ Department of Veterinary Pharmacology, Graduate School of Agriculture and Life Sciences, The University of \\ Tokyo, Tokyo, Japan
}

*Corresponding author: Md. Shafiqul Islam, D.V.M., Ph.D., Department of Pharmacology, Bangladesh Agricultural University, Mymensingh-2202, Bangladesh. E-mail: shafiqpharma@yahoo.co.uk

Received: 07 November 2016/Accepted: 13 November 2016/ Published: 29 December 2016

\begin{abstract}
Interlukin-33 (IL-33) is a novel cytokine of the IL-1 superfamily that is expressed mainly in stromal cells, such as epithelial and endothelial cells. It is believed to be involved in inflammation and carcinogenesis. Recently we have reported that IL-33 is up regulated in azoxymethane-dextran sulfate sodium salt (AOM/DSS)induced in colitis in mice. In this study we have investigated the biological activity of IL-33 in cells migration in IEC-6 epithelial cells. Our wound healing assay demonstrated that IL-33 significantly enhanced cell migration towards the wound edges compared with the untreated control cells. Trans-well migration assay in presence of cells culture and wound stimulation in the lower chamber, IL-33 exhibited significant chemoattractant activity for the cells migration. On the other hand, in absence of cells culture and wound stimulation in the lower chamber, IL-33 neither produced remarkable chemokine, nor chemoattractant activity for the cells migration. These findings indicate that IL-33 interacts with wound-stimulated soluble cellular product(s) in the lower chamber to accelerate cell migration.
\end{abstract}

Keywords: IEC-6 intestinal epithelial cell; interleukin-33; migration; wound healing

\section{Introduction}

Interlukin-33 (IL-33) is a novel a member of IL-1 family of cytokines with a growing number of target cells and a plethora of biological functions (Martin et al., 2016). IL-33 is expressed mainly in stromal cells, such as epithelial and endothelial cells (Miller, 2011). IL-33 doesn't express it function like typical cytokines, it exerts biological effects through activation of the membrane associated ST2 receptor (Yagami et al., 2010) and as an intracellular nuclear factor with transcriptional regulatory properties (Haraldsen et al., 2009; Kakkar et al., 2008). Although IL-33 was previously described as a nuclear factor, it is expressed both in the cytoplasm and nucleus. IL-33 is now thought to function as an alarmin that is released following cell necrosis to alert the immune system to tissue damage or stress ( Pichery et al., 2012). Now a day, special emphasis has been given on the pro-inflammatory roles of the IL-33/ST2 signaling pathway, particularly in gastrointestinal pathophysiology related to colitis and carcinogenesis. Recently it is reported that active ulcerative colitis (UC) patients express significantly elevated levels of IL-33 mRNA and sub-epithelial myofibroblasts act as a major source of IL-33(Kobori et al., 2010) and play a critical role in mucosal repair process in the mucosa of UC patients (Andoh et al., 2002).

Cell migration is an important phenomenon involved both in innate and adaptive immunity in the body. Inflammation depends on the migration and recruitment of varieties of immune cells at the site of inflammation. Recent evidences suggested that IL-33 is a selective Th2 chemoattractant associated with pro-inflammatory activity (Komai-Koma et al., 2007). IL-33 accelerated the invasion and migration of gastric cancer cells, while, 
knockdown of the IL-33 receptor ST2 attenuated the IL-33-mediated invasion and migration (Yu et al., 2015). Recently a group of scientist reported that IL-33-ST2 promotes metastasis through the interaction between perivascular cells and tumor-associated macrophages in xenograft mouse models of cancer (Yang et al., 2016). Considering the above backgrounds, we aimed to study the biological activities of IL-33 in cell migration, if any.

\section{Materials and Methods}

\subsection{Cell culture}

The IEC-6 rat small intestinal cell line was obtained from the RIKEN Cell Bank (RCB0993, Japan). The cells were cultured in DMEM medium containing 10\% FBS. The condition of the cells was observed using phase contrast microscopy and healthy cells were sub-cultured for subsequent experimentation. In all cases, the experiments were performed in at least triplicate.

\subsection{Wound healing assay}

A wound healing assay was performed using IEC-6 cells (Islam et al., 2014). IEC-6 cells were cultured in 6well plates until confluence and then serum-starved overnight. Thereafter, the confluent cell layer was scratched with a pipette tip and the medium was changed. Images (4fields/well) were acquired of the scratched cells, which were then washed and treated with medium containing IL-33 (30 ng/mL). After 24 hours, new images were acquired of the same fields and wound areas were measured using Image $\mathbf{J}$ software. Five wells per condition were examined.

\subsection{Trans-well migration assay}

Trans-well chambers ( $8-\mu$ m pore size, BD Biosciences, Durham, NC, USA) were prepared by pre-coating with gelatin $(1 \%)$. IEC-6 cells $\left(5 \times 10^{5}\right)$ in $300 \mu$ of DMEM were placed in the upper chamber of cell culture inserts in a 24-well plate. In the lower chambers, IEC-6 cells were cultured and stimulated with wound stimulation, as above. FBS $(1 \%)$ or IL-33 (30 ng/mL) was added to the lower chamber and IL-33 $(30 \mathrm{ng} / \mathrm{mL})$ was added to the upper chamber. The cells were incubated for 8 hours and cell migration was enumerated in five randomly selected fields for each experiment. Similarly, experiments were also performed in the absence of cells in the lower chamber. The upper chamber of the cell culture insert was placed into the lower chamber. FBS (1\%) or IL-33 (30 ng/mL) was added to the lower chamber and IL-33 (30 ng/mL) was added to the upper chamber. The cells were incubated for 8 hours and cell migration was enumerated in five randomly selected fields for each experiment.

\subsection{Statistical analysis}

All experiments were performed with a minimum of triplicate replication, and values are expressed as mean \pm SEM. Statistical significance $\left(* P<0.05\right.$ and $\left.{ }^{* *} P<0.01\right)$ was determined by using the Student's $t$ test and oneway ANOVA, as appropriate.

\section{Results}

\subsection{IL-33 promotes epithelial cells migration in wound healing assay}

Cell migration is an important inherent qualities of live cells and critical for normal development, immune response, and disease processes such as cancer metastasis and inflammation. In this study, biological activity of IL-33 was investigated in concept of cell migration potentiality in wound healing assays. IEC- 6 cells were cultured and serum-starved overnight, and the monolayer was wounded by scratching with a pipette tip. The cells then washed with HBSS, imaged (0 hour) and the medium replaced (DMEM containing $30 \mu \mathrm{g} / \mathrm{mL}$ IL-33). After 24 hours, the IL-33-treated cells showed significant enhanced cell migration towards the wound edges compared with the untreated control cells (Figure 1 A-B). 


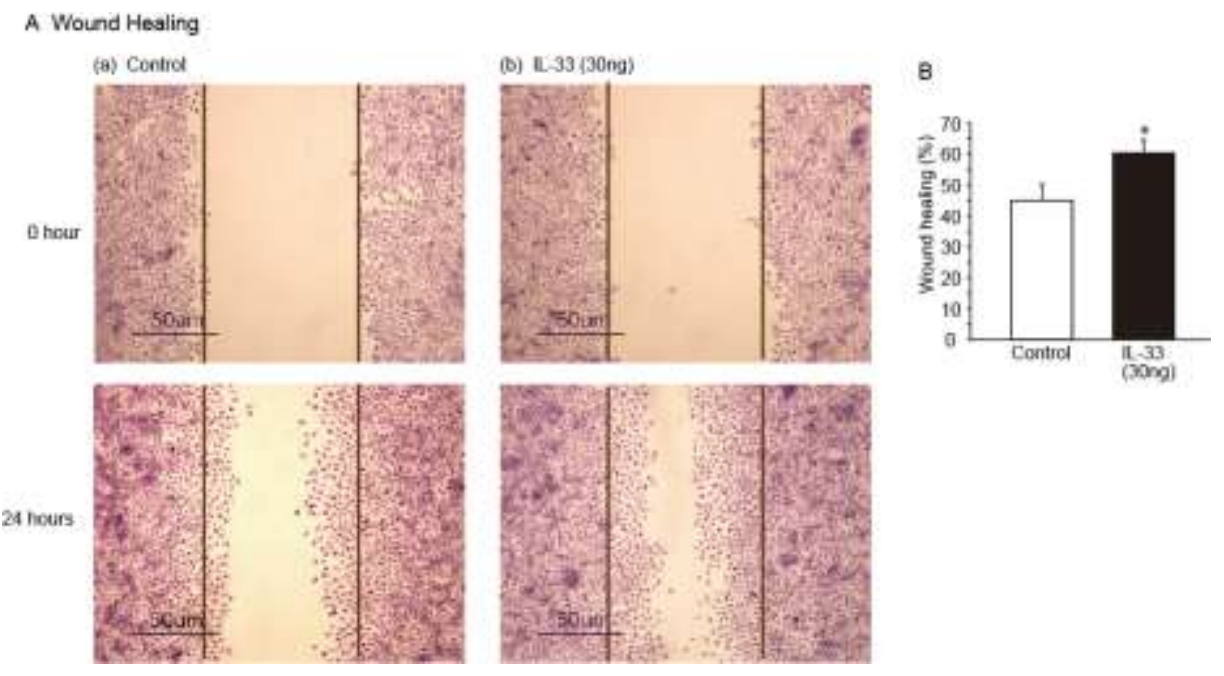

Figure 1. IL-33 enhances IEC cell migration in a wound-healing assay. IEC-6 cells were cultured, serumstarved overnight, scratched, and treated with IL-33 (30 ng/mL) for 24 hours. A: (a) the image shows cell migration in n non-treated control; (b) the image shows enhanced cell migration in IL-33-treated cells. B: Analysis of cells migration indicates that IL-33 significantly increases cell migration at 24 hours. *P<0.05; significantly different from control $(n=5)$.

\subsection{IL-33 promotes epithelial cells migration in trans-well migration assay}

Trans-well migration assay is an important tool to assess the potentiality of any chemicals or drugs or inherent properties that accelerates or inhibits cell migration during physiological and pathological stages of the body. The role of IL-33 on the migration of IEC-6 cells was assessed using a trans-well assay, in the absence (no cell culture) and presence of IEC- 6 cells in the lower chamber. In the absence of IEC- 6 cells in the lower chamber IL-33 $(30 \mathrm{ng} / \mathrm{mL})$ added to either the upper or lower chambers produced little or no chemoattractant activity for cell migration (Figure 2A-B). On the other hand, IEC-6 cells were cultured to confluence in the lower chamber prior to wound stimulation as like wound healing assay. We found that the inclusion of wounded IEC- 6 cells in the lower chamber resulted in IL-33 $(30 \mathrm{ng} / \mathrm{mL})$ inducing cell migration by exerting chemoattractant activity (Figure 3A-B). These findings indicate that IL-33 interacts with wound-stimulated soluble cellular product(s) in the lower chamber to accelerate cell migration. It is to be mentioned that in both techniques, $1 \% \mathrm{FBS}$ in the lower chamber showed a high chemoattractant activity in trans-well migration assay.

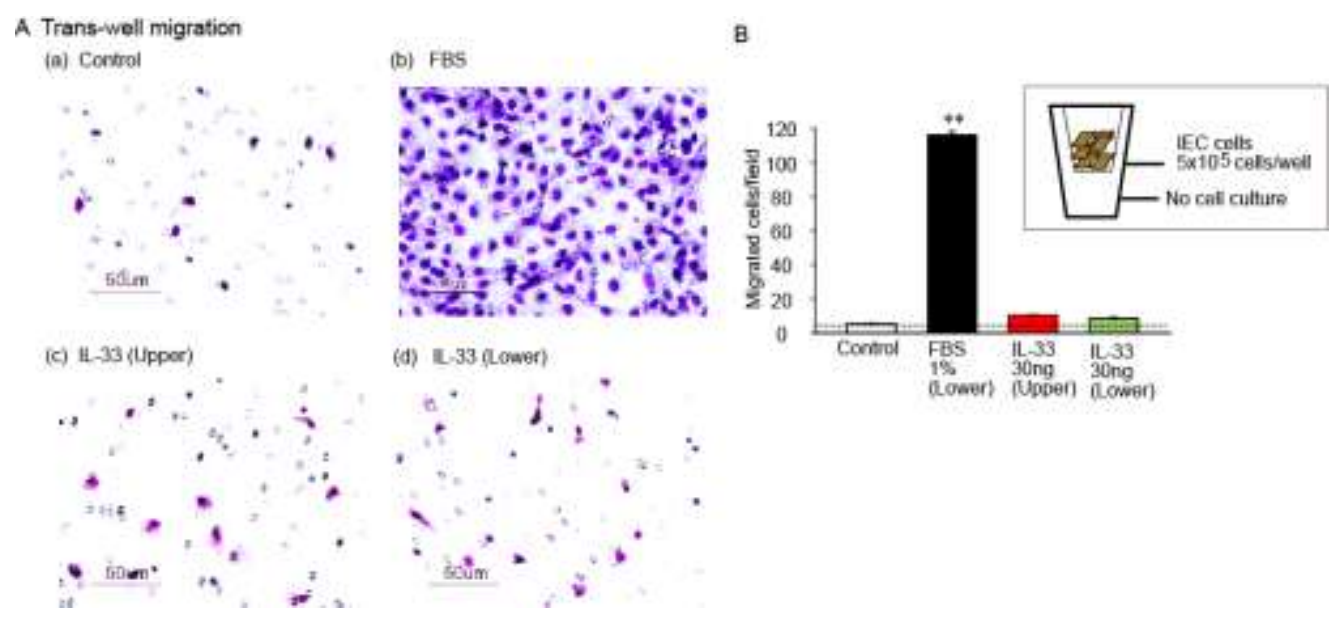

Figure 2. Effects of IL-33 on cell migration in IEC-6 cells. A: IEC-6 cells were allowed 8 hours to migrate through the trans-well membranes. A: (a) Control (no treatment); (b) FBS 1\% (Lower); (c) IL-33 30 $\mathrm{ng} / \mathrm{mL}$ (Upper) and (d) IL-33 $30 \mathrm{ng} / \mathrm{mL}$ (Lower). Bar=50 $\mathrm{mm}$. B, Analytical data indicate that only FBS increased cell migration at 8 hours. $* * P<0.01$; significantly different from control $(n=4)$. Schematic representation of the trans-well migration assay experimental design. 


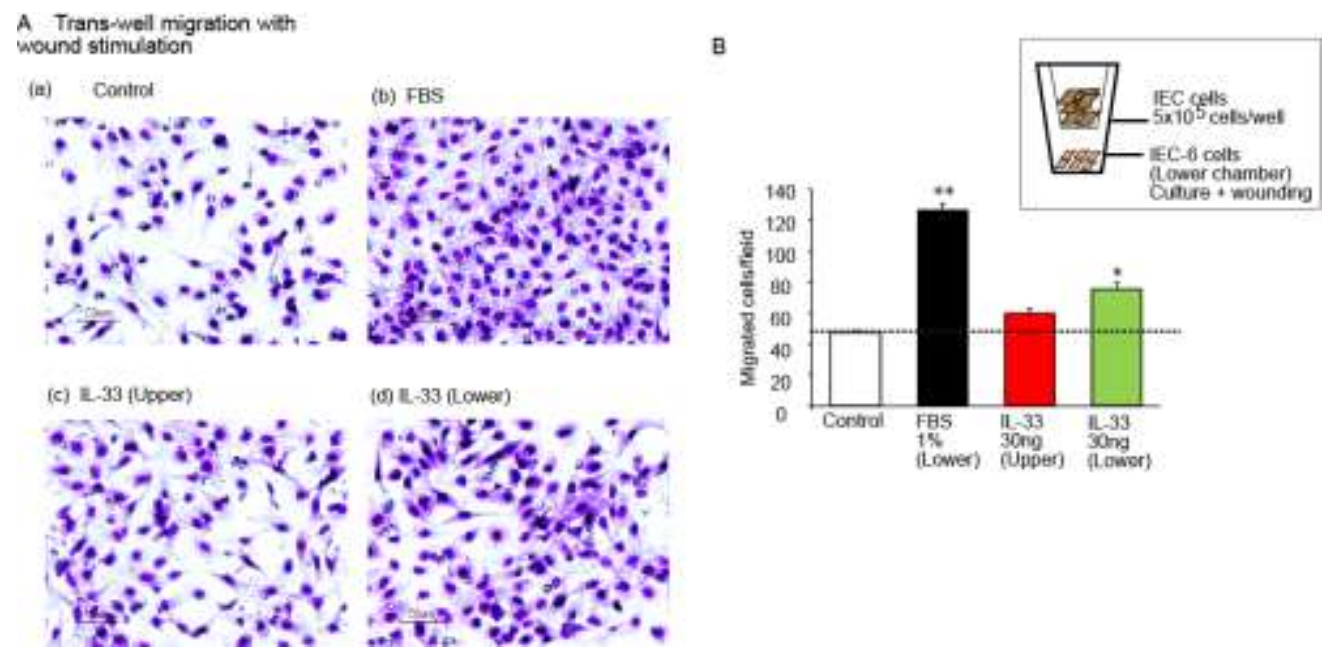

Figure 3. IL-33 enhances IEC cell migration in a wound-stimulation dependent manner in a trans-well migration assay. A: IEC-6 cells were allowed 8 hours to migrate through the trans-well membranes. (a) Control (no treatment), (b) FBS 1\% (Lower), (c) IL-33 $30 \mathrm{ng} / \mathrm{mL}$ (Upper) and (d) IL-33 30 ng/mL (Lower). Bar $=50 \mu \mathrm{m}$. B, Analytical data indicate that only FBS increased cell migration at 8 hours. $* P<0.05$ and $* * P<0.01$; significantly different from control $(\mathrm{n}=4)$. Schematic representation of the transwell migration assay experimental design.

\section{Discussion}

Migration is a key property of live cells and critical for normal development, immune response, and disease processes such as cancer metastasis and inflammation (Justus et al., 2014). Methods to examine cell migration are very useful and important for a wide range of biomedical research such as cancer biology, immunology, vascular biology, cell biology and developmental biology (Justus et al., 2014). The well-known intestinal epithelial cell line (IEC-6 cells) was chosen to study the biological effects of IL-33 in cell migration assay. The pharmacological action of IL-33 in a wound-healing assay was then assessed. Treatment of IEC-6 cells with IL33 for 24 hours produced enhanced cell migration in the wound-healing assay. Wound stimulation is a pathological process that involves many inflammatory signaling pathways. Therefore, we investigated the physiological role of IL-33 in cell migration. We used two trans-well migration assay methods, the conventional method with no cells in the lower chamber and an alternative method that involved IEC-6 cell culture in the lower chamber followed by wound stimulation. In the absence of cells in the lower chamber, application of IL33 to either the upper or lower chamber resulted in little or no chemoattractant activity and cell migration. However, in the presence of wounded cells in the lower chamber, application of IL-33 to the lower chamber resulted in elevated chemoattractant activity and cell migration. These findings suggest that IL-33 interacts with wound-stimulated soluble cellular product(s) present in the lower chamber that influence the rate of cell migration. Others have reported that IL-33 is a powerful chemoattractant molecule, in both in vitro and in vivo experiments, with pro-inflammatory properties (Komai-Koma et al., 2007). There is a scientific argument that following wound stimulation, cells release many soluble products into the medium, including known and unknown factors released from dead or damage cells that could interact with IL-33 and elicit a complex signaling phenomenon.

\section{Conclusions}

The increased IL-33 production during inflammation contributes to epithelial cell migration, remodeling, and repair of damaged areas or metastasis in the cancer environment.

\section{Conflict of interest}

None to declare.

\section{References}

Andoh A, S Fujino, T Okuno,Y Fujiyama and T Bamba, 2002. Intestinal subepithelial myofibroblasts in inflammatory bowel diseases. J. Gastroenterol., 14: 33-37. 
Haraldsen G, J Balogh, J Pollheimer, J Sponheim and AM Kuchler, 2009. Interleukin-33 - cytokine of dual function or novel alarmin? Trends Immunol., 30: 227-233.

Islam MS, M Kusakabe, K Horiguchi, S Iino, T Nakamura, K Iwanaga, H Hashimoto, S Matsumoto, T Murata, M Hori and $\mathrm{H}$ Ozaki, 2014. PDGF and TGF-beta promote tenascin-C expression in subepithelial myofibroblasts and contribute to intestinal mucosal protection in mice. Br. J. Pharmacol., 171: 375-388.

Justus CR, N Leffler, M Ruiz-Echevarria and LV Yang, 2014. In vitro cell migration and invasion assays. Journal of Visualized Experiments, JoVE(88).

Kakkar R and RT Lee, 2008. The IL-33/ST2 pathway: therapeutic target and novel biomarker. Nat. Rev. Drug Discov., 7: 827-840.

Kobori A, Y Yagi, H Imaeda ,H Ban, S Bamba, T Tsujikawa, Y Saito, Y Fujiyama and A Andoh, 2010. Interleukin-33 expression is specifically enhanced in inflamed mucosa of ulcerative colitis. J. Gastroenterol., 45: 999-1007.

Komai-Koma M, D Xu , Y Li, AN McKenzie , IB McInnes and FY Liew, 2007. IL-33 is a chemoattractant for human Th2 cells. Europian Journal of Immunology, 37: 2779-2786.

Lloyd CM, 2010. IL-33 family members and asthma - bridging innate and adaptive immune responses. Curr. Opin. Immunol., 22: 800-806.

Martin NT and MU Martin, 2016. Interleukin 33 is a guardian of barriers and a local alarmin. Nat. Immunol., 17: 122-131.

Miller AM, 2011. Role of IL-33 in inflammation and disease. Journal of Inflammion, 8: 22.

Pichery M, E Mirey, P Mercier, E Lefrancais, A Dujardin , N Ortega and JP Girard, 2012. Endogenous IL-33 is highly expressed in mouse epithelial barrier tissues, lymphoid organs, brain, embryos, and inflamed tissues: in situ analysis using a novel Il-33-LacZ gene trap reporter strain. J. Immunol., 188: 3488-3495.

Yagami A, K Orihara, H Morita, K Futamura, N Hashimoto, K Matsumoto,H Saito and A Matsuda, 2010. IL33 mediates inflammatory responses in human lung tissue cells. J. Immunol., 185: 5743-5750.

Yang Y, P Andersson, K Hosaka, Y Zhang, R Cao, H Iwamoto, X Yang, M Nakamura, J Wang, R Zhuang, H Morikawa, Y Xue, H Braun, R Beyaert, N Samani, S Nake, E Hams, S Dissing, PG Fallon, R Langer and Y Cao, 2016. The PDGF-BB-SOX7 axis-modulated IL-33 in pericytes and stromal cells promotes metastasis through tumour-associated macrophages. Nat. Commun., 7: 11385.

Yu XX, Z Hu, X Shen, LY Dong, WZ Zhou and WH Hu, 2015. IL-33 Promotes Gastric Cancer Cell Invasion and Migration Via ST2-ERK1/2 Pathway. Digestive Diseases and Sciences, 60: 1265-1272. 